\title{
Cue-evoked dopamine release in the nucleus accumbens shell tracks reinforcer magnitude during intracranial self-stimulation
}

\author{
Manna Beyene ${ }^{1}$, Regina M. Carelli ${ }^{1,2,3}$, and R. Mark Wightman ${ }^{1,3,4}$ \\ ${ }^{1}$ Curriculum in Neurobiology, University of North Carolina at Chapel Hill, Chapel Hill, North \\ Carolina 27599-3290 \\ 2Department of Psychology, University of North Carolina at Chapel Hill, Chapel Hill, North \\ Carolina 27599-3290 \\ ${ }^{3}$ Neuroscience Center, University of North Carolina at Chapel Hill, Chapel Hill, North Carolina \\ 27599-3290 \\ ${ }^{4}$ Department of Chemistry, University of North Carolina at Chapel Hill, Chapel Hill, North Carolina \\ 27599-3290
}

\begin{abstract}
The mesolimbic dopamine system is critically involved in modulating reward-seeking behavior and is transiently activated upon presentation of reward-predictive cues. It has previously been shown, using fast-scan cyclic voltammetry in behaving rats, that cues predicting a variety of reinforcers including food/water, cocaine or intracranial self-stimulation (ICSS) elicit time-locked transient fluctuations in dopamine concentration in the nucleus accumbens (NAc) shell. These dopamine transients have been found to correlate with reward-related learning and are believed to promote reward-seeking behavior. Here, we investigated the effects of varying reinforcer magnitude (intracranial stimulation parameters) on cue-evoked dopamine release in the NAc shell in rats performing ICSS. We found that the amplitude of cue-evoked dopamine is adaptable, tracks reinforcer magnitude and is significantly correlated with ICSS seeking behavior. Specifically, the concentration of cue-associated dopamine transients increased significantly with increasing reinforcer magnitude, while, at the same time, the latency to lever press decreased with reinforcer magnitude. These data support the proposed role of NAc dopamine in the facilitation of rewardseeking and provide unique insight into factors influencing the plasticity of dopaminergic signaling during behavior.
\end{abstract}

\section{Keywords}

in vivo voltammetry; carbon-fiber microelectrode; cue-evoked dopamine release

\section{Introduction}

The mesolimbic dopamine system is believed to play an essential role in modulating goaldirected behaviors and has been implicated in reinforcement learning (Hollerman and

\footnotetext{
(C) 2010 IBRO. Published by Elsevier Ltd. All rights reserved.

Corresponding Author: Dr. R. Mark Wightman Phone: 919-962-1472 Fax: 919-962-2388 rmw@unc.edu.

Publisher's Disclaimer: This is a PDF file of an unedited manuscript that has been accepted for publication. As a service to our customers we are providing this early version of the manuscript. The manuscript will undergo copyediting, typesetting, and review of the resulting proof before it is published in its final citable form. Please note that during the production process errors may be discovered which could affect the content, and all legal disclaimers that apply to the journal pertain.
} 
Schultz, 1998, Dayan and Balleine, 2002, Day et al., 2007, Owesson-White et al., 2008). Electrophysiological data have shown that midbrain dopamine neurons are transiently activated by the delivery of unexpected, primary rewards and also by cues that predict rewards (Schultz et al., 1997, Hyland et al., 2002). Similarly, data from fast-scan cyclic voltammetric recordings have revealed that cues associated with a variety of rewards, including cocaine, sucrose, and intracranial stimulation elicit time-locked dopamine transients in the NAc (Phillips et al., 2003b, Roitman et al., 2004, Day et al., 2007, Owesson-White et al., 2008). Indeed, the fluctuations in dopamine concentration in the extracellular fluid of the NAc are a direct consequence of increased phasic activity in the ventral tegmental area (VTA) (Sombers et al., 2009). The dopaminergic signal resulting from the activation of dopamine neurons is thought to then guide decision making and modulate reward-seeking behavior (Goto and Grace, 2005, Nicola et al., 2005, Morris et al., 2006).

The degree of dopamine activation in response to reward-predictive cues has been found to reflect salient information about predicted rewards, including the probability and timing of their occurrence (Fiorillo et al., 2003, Kobayashi and Schultz, 2008). Furthermore, electrophysiology studies have shown that cues predicting larger reinforcer magnitudes elicit greater activation of dopamine neurons than cues that predict smaller reinforcer magnitudes, suggesting that dopamine neurons encode important information related to the amount of impending rewards (Tobler et al., 2005, Roesch et al., 2007). These findings have recently been supported by measurements of transient dopamine release events in the NAc core during reward presentation (Gan et al., 2010). However, it remains to be shown whether these adaptive changes in neural activation also manifest themselves in the NAc shell. This is particularly important since recent anatomical data show that distinct subregions of the VTA send differential projections to particular subregions of the NAc (Ikemoto, 2007), and a one-to-one correspondence between VTA dopamine cell firing and dopamine release is not always evident (Montague et al., 2004, Kita et al., 2007).

Here, we used fast-scan cyclic voltammetry at carbon-fiber microelectrodes to examine the effect of reinforcer magnitude on cue-evoked dopamine release in the shell of the NAc, a key dopamine terminal region. This electrochemical technique enables detection of dopamine release with subsecond temporal resolution (Robinson et al., 2003) in 'real-time' during behavior. The behavioral paradigm employed is intracranial self-stimulation (ICSS), in which a rat depresses a lever to electrically stimulate select brain pathways (Wise, 2004). We have previously shown that cue-evoked dopamine release in the NAc shell is associated with ICSS and that the cue-evoked signal develops with learning (Owesson-White et al., 2008). Here we show that the concentration of cue-associated dopamine transients increases significantly with increasing reinforcer (current) magnitude. Furthermore, increases in cueevoked dopamine were significantly correlated with shorter latencies to lever press, further establishing a role for dopamine in the facilitation of learned, reward-seeking behavior (Nicola et al., 2005).

\section{Methods}

\section{Surgical Procedures}

Male, Sprague-Dawley rats were individually housed on a 12:12 hour light:dark cycle and allowed ad libitum access to food and water. Animals were anesthetized with intraperitoneal injections of ketamine $(100 \mathrm{mg} / \mathrm{kg})$ and xylazine $(20 \mathrm{mg} / \mathrm{kg})$. Bupivicaine was used as a local analgesic. Stereotaxic surgeries were performed using aseptic, flat-skull technique using coordinates from a stereotaxic atlas (Paxinos and Watson, 2007). Surgical procedures have been described previously (Phillips et al., 2003a). Briefly, guide cannulae (Bioanalytical Systems, West Lafayette, IN) were cut to $2.5 \mathrm{~mm}$ and implanted above the 
NAc shell (1.7 mm anterior, $0.8 \mathrm{~mm}$ lateral, relative to bregma). Bipolar, stainless-steel stimulating electrodes (Plastics One, Roanoke, VA) were ipsilaterally implanted in the lateral hypothalamus ( $2.8 \mathrm{~mm}$ posterior, $1.7 \mathrm{~mm}$ lateral relative to bregma, and $8.4 \mathrm{~mm}$ ventral from dura) and $\mathrm{Ag} / \mathrm{AgCl}$ reference electrodes were placed in the contralateral hemisphere. Skull screws and cranioplastic cement were used to secure electrode placements. Animals were given at least 3 days to recover. These procedures were in accordance with guidelines set forth by The University of North Carolina at Chapel Hill Animal Care and Use Committee.

\section{Behavioral Procedures}

Animals were placed in an operant chamber and given 5 min to acclimate. They were then attached, via a flexible cable, to a commutator secured to the top of the chamber that allowed for both voltammetric recording and electrical stimulation. An additional acclimation period of $5 \mathrm{~min}$ was given before the first phase of ICSS training commenced in which rats were trained to press a continuously available lever on a fixed-ratio 1 (FR1) reinforcement schedule for ICS. In all experiments described here, each lever press resulted in the delivery of a $60 \mathrm{~Hz}$ stimulation ( 24 biphasic pulses, $2 \mathrm{~ms}$ for each phase). The stimulation was delivered within 0.1-0.2 s after the lever press. During initial training phase the current was $100-150 \mu \mathrm{A}$, a current range found to promote ICSS learning (OwessonWhite et al., 2008). Once rats exhibited stable responding (30 consecutive lever presses), the session was stopped, the lever was retracted, and the next phase of training commenced.

Next, a threshold curve was determined for each animal $(n=6)$ to establish their low, medium and high reinforcer magnitude levels (Gallistel and Leon, 1991, Gallistel et al., 1991, Simmons and Gallistel, 1994, Arvanitogiannis and Shizgal, 2008). This was accomplished by measuring the number of reinforced presses in separate 1 min intervals for different stimulation currents. Specifically, each rat was given free access to the lever that remained extended throughout this phase. Stimulation currents were varied randomly between intervals and ranged from 16 to $200 \mu \mathrm{A}$ in $0.1 \log$ unit increments while the number of lever presses at each current was recorded. For each animal, medium magnitudes were set at currents that produced maximal responding. Currents that produced half-maximal responding to the left and right of the maximum were chosen as the low and high magnitudes, respectively. In this manner, a low, medium, and high magnitude was established for each individual animal for further evaluation in the VTO paradigm.

Rats were then trained to perform ICSS on an FR1 with a variable intertrial interval. The variable time-out (VTO, the times were normally distributed values between 5 and $25 \mathrm{~s}$; longer intervals led to difficulty in maintaining the behavior) procedure was identical to that described previously (Owesson-White et al., 2008). During the first 50 trials, lever extension was accompanied by simultaneous presentation of an audio-visual cue (a $67 \mathrm{~dB}, 1 \mathrm{kHz}$ tone coupled with a change in the lighting of the experimental chamber). Depression of the lever delivered the stimulus train (the current during this training was at the medium level for the particular animal). For the next 150 trials, the audio-visual cue preceded lever extension by $2 \mathrm{~s}$. When training for the VTO schedule was complete, a fresh carbon-fiber microelectrode was lowered into the NAc shell for voltammetric recording and an optimal recording site was identified (see below). The animal was then allowed to perform ICSS on the same VTO schedule with the audio-visual cue preceding lever extension by $2 \mathrm{~s}$. Each rat was given three distinct and consecutive ICSS sessions comprised of 70 trials that used low, medium, or high stimulation currents. These sessions were randomly ordered (i.e. low-medium-high, low-high-medium, etc) and were separated by 5 minutes. The same audio-visual cue was used for all stimulation currents. After the recording sessions the animals were sacrificed. 


\section{Voltammetric Recording}

Microelectrodes were prepared by aspirating T650 carbon fibers ( $6 \mu \mathrm{m}$ diameter, Amoco) into thin glass capillaries ( $0.6 \mathrm{~mm}$ outer diameter, $0.4 \mathrm{~mm}$ inner diameter, A-M Systems). Filled glass capillaries were then pulled in a vertical puller (Narishige, Tokyo, Japan) and the exposed carbon fiber tip was cut to $75 \mu \mathrm{m}-100 \mu \mathrm{m}$. For electrochemical recordings, a triangular waveform $(-0.4 \mathrm{~V}$ to $+1.3 \mathrm{~V}$ vs. $\mathrm{Ag} / \mathrm{AgCl})$ was applied at $400 \mathrm{~V} / \mathrm{s}$ to the carbonfiber microelectrode every $100 \mathrm{~ms}$. Optimal recording sites within the NAc shell were sought by incrementally lowering the electrode through the NAc and measuring electrically evoked dopamine release, ensuring placement in an area rich in active dopamine terminals. After use, electrodes were calibrated in an in vitro flowcell system to enable conversion of dopamine current measurements into concentration units. Principal component regression was used to resolve recorded dopamine signals from interfering species, namely $\mathrm{pH}$ (Heien et al., 2004, Heien et al., 2005). Amplitudes of released dopamine were characterized using MiniAnalysis software (Synaptosoft, version 6.03).

\section{Data Analysis}

Maximal cue and stimulated mean dopamine concentrations were compared across low medium, and high trials using a repeated measures one-way Analysis of Variance (ANOVA), followed by Tukey's post hoc test. Linear regression was used to evaluate maximal dopamine concentration changes as a function of stimulation current for both stimulated and cue-evoked release. Linear regression was also used to evaluate the relationship between latency to lever press and the amplitude of maximal cue evoked dopamine concentration. Statistical significance was set to $\mathrm{p}<0.05$.

\section{Results \\ Determination of 'Low', 'Medium' and 'High' current parameters for ICSS using the threshold curve procedure}

As shown in Figure 1, stimulation current amplitude was found to play an important role in lever pressing behavior during ICSS. When given free access to a permanently extended lever, lever presses were infrequent at stimulation currents of $40 \mu \mathrm{A}$ or less. The lever pressing rate increased at $50 \mu \mathrm{A}$ and reached a plateau at higher stimulation currents. At the highest current evaluated, $200 \mu \mathrm{A}$, lever pressing rate decreased. However, the decrease in lever press rate at this current was accompanied by a strong physical reaction of the rat to the stimulation. Reflexively, the animal moved away from the lever after each press. Based on these data we selected three stimulation intensities for each individual animal for further evaluation using the VTO paradigm. The average stimulation currents used in all animals were $67 \mu \mathrm{A} \pm 8.71,115 \mu \mathrm{A} \pm 11.4$, and $186.67 \mu \mathrm{A} \pm 8.43$, which were defined as low, medium, and high, respectively.

\section{Dynamic changes in dopamine release occur in response to the reward-predictive cue during ICSS}

Following the threshold curve procedure, cue- and stimulation-evoked dopamine responses were determined using fast-scan cyclic voltammetry during ICSS for the low, medium and high currents on the VTO schedule. The time course of the VTO reinforcement schedule is given in Figure 2A (top). Each trial began with the onset of an audio-visual cue that preceded lever extension by $2 \mathrm{~s}$. Once the lever was pressed, it retracted, the stimulation was delivered and the audio-visual cue was terminated. Trials were separated by a VTO of $5 \mathrm{~s}-$ $25 \mathrm{~s}$. As shown in the representative color plot from a single trial in a well-trained animal, cue onset elicited a time-locked increase in dopamine release (Figure 2A, center). Electrically stimulated dopamine release was also readily observed immediately following 
the lever press. A corresponding trace of the dopamine concentration with time (resulting from principal component regression of these data) is shown for this trial just below the color plot (Figure 2A, bottom).

For each animal, individual trials within each reinforcer magnitude session were averaged to examine the mean dopaminergic response in each condition. An average electrochemical signal observed in a high reinforcer magnitude session for one animal is shown in a representative color plot in Figure 2B. The corresponding concentration trace for dopamine is also displayed. Cue-evoked dopamine release occurred in a tightly time-locked fashion with the initial rise beginning directly after cue onset. Electrically evoked dopamine release was observed following the lever press. Thus, consistent with our prior reports, a transient, time-locked increase in dopamine concentration occurs in the NAc shell upon presentation of a cue that predicts ICSS availability (Owesson-White et al., 2008, Sombers et al., 2009).

\section{Adaptations in cue-evoked dopamine release closely follow transitions in reinforcer magnitude}

The amplitude of cue-evoked dopamine release in the NAc reflects the magnitude of the predicted reinforcer (Figure 3). Figure 3A displays representative average dopamine concentration traces for the low, medium, and high reinforcer magnitude sessions from a single animal. Individual trial maximal concentration values averaged across all animals are shown in Figure 3B. Note that the amount of dopamine released, both in response to cue onset (Figure 3B, upper panels) and the electrical stimulation (Figure 3B, lower panels), increases significantly with increasing reinforcer magnitude (cue-evoked dopamine: High $>$ Medium > Low, $\mathrm{p}<0.001$, one-way ANOVA $F(2,59)=1.38$; stimulated dopamine: High > Medium > Low, $\mathrm{p}<0.001$ oneway ANOVA $F(2,69)=1.80)$. The mean \pm SEM values for cue-evoked and stimulated dopamine release are given in Table 1.

For each animal, the average maximal concentration for cue- and stimulation-evoked dopamine release was determined at each stimulation intensity. Dopamine concentrations are plotted against stimulus current intensity in Figure 4A. Both stimulated release and cueevoked dopamine release increase linearly with stimulation current, but with slopes that are significantly different $(\mathrm{p}<0.001)$.

\section{Cue-evoked dopamine release in the NAc is inversely correlated with latency to press during ICSS}

In addition to modulating rapid dopamine signaling, reinforcer magnitude also significantly affected the average latency to lever press for ICSS. The average latency to press decreased significantly with increasing reinforcer magnitude (low $>$ medium, $\mathrm{p}<0.001$, medium $>$ high, $\mathrm{p}<0.05$, one-way ANOVA $F(2,69)=1.28$, Table 1$)$. Moreover, the latency to lever press was found to significantly correlate with the amplitude of cue-evoked dopamine release in an inverse manner $\left(r^{2}=0.337, p=0.023\right.$, Figure $\left.4 B\right)$. Thus, higher levels of cueassociated dopamine were correlated with shorter latencies to press and vice versa.

\section{Discussion}

Reward-predictive cues activate midbrain dopaminergic neurons and elicit time-locked, transient increases in dopamine release in terminal regions (Schultz et al., 1997, Hyland et al., 2002, Phillips et al., 2003b, Roitman et al., 2004, Day et al., 2007, Owesson-White et al., 2008). Furthermore, the degree of activation of these dopaminergic neurons has been found to vary with the magnitude of the anticipated reinforcer (Tobler et al., 2005). Cues that predict larger reinforcer magnitudes produce more pronounced activation of dopamine neurons than cues that predict smaller reinforcer magnitudes (Tobler et al., 2005, Roesch et 
al., 2007). In the NAc core, the amplitude of cue-induced dopamine concentration transients scales with the magnitude of an anticipated reward (Gan et al., 2010). Thus, dopamine neurons appear to be conditional output neurons that reflect salient reward-related information in their patterns of activity. Here, we show that the amplitude of cue-evoked dopamine release in the NAc shell, a key dopamine terminal region, reflects impending reinforcer magnitude. We also found a significant correlation between the amplitude of cueevoked dopamine release in the NAc shell and the latency to lever press for ICSS. Together, our results provide unique insight into the adaptable nature of dopaminergic signaling in a key terminal region and demonstrate an integral role of cue-evoked dopamine in rewardseeking behavior.

Discovered in 1954, ICSS has been widely used to investigate goal-directed behavior and to identify brain structures involved in mediating reinforcement (Olds and Milner, 1954, Olds and Olds, 1963, Simon et al., 1975, Corbett and Wise, 1980, Wise, 1996, Waraczynski, 2006). In this behavioral paradigm, animals are trained to perform an operant response, typically a lever press, to deliver an electrical stimulation to select brain areas. An advantage of ICSS is that it directly activates neural circuits involved in reward, bypassing normal physiological inputs (Wise, 1996). Thus, the precise timing and magnitude of reinforcement delivery can be tightly controlled and measured. Several pioneering studies in the field have shown that the magnitude of experienced reinforcement during ICSS is a function of both stimulation current and pulse frequency (Gallistel and Leon, 1991, Gallistel et al., 1991, Simmons and Gallistel, 1994, Arvanitogiannis and Shizgal, 2008). It has been hypothesized that the maximum possible reward during ICSS can be attained by manipulating stimulation current (Waraczynski and Kaplan, 1990, Gallistel et al., 1991, Sax and Gallistel, 1991).

Indeed, using a threshold curve procedure we found that stimulation current played an influential role in lever pressing behavior. This approach enabled assessment of relative reinforcer value for each animal. As shown in the threshold curve in Figure 1, the response rate for ICSS was initially relatively low at the low stimulation currents and peaked at $\sim 125 \mu \mathrm{A}$ before declining. However, the decline in lever press rate at the highest current evaluated appeared to be controlled in part by the animals' reflexive response to the stimulation and not to a decrease in the reinforcing properties of the stimulation. To examine this possibility, we used latency to lever press as a measure of reinforcer value (i.e., animals respond faster for a more valued reinforcer). Indeed, the significant difference in the latency to lever press between the high and the medium reinforcer magnitude conditions, observed with the variable time-out schedule, indicates that the subjective reward value for the high reinforcer magnitude was in fact higher (see Table 1).

With the VTO paradigm we have shown that, in a well-trained animal, presentation of an audio-visual cue that predicts ICSS produces a transient increase in dopamine concentration in the NAc shell. The association between transient dopamine release and cues that predict lever availability develops in the NAc shell with repeated trials during acquisition of ICSS (Owesson-White et al., 2008). This increase in cue-evoked dopamine concentration was correlated with learning of the behavior, indicated by a significant decrease in the latency to lever press in early trials, and is well learned after 200 trials. In addition to developing with repeated stimulus-reward pairings during the course of learning, cue-evoked dopamine release disappears within one trial during extinction of ICSS indicating that cue-evoked dopamine release is dependent on a learned stimulus-reward association and is reinstated just as rapidly (Owesson-White et al., 2008). In the NAc core, cue evoked release is not seen on early trials (Cheer et al., 2007), and it develops at a slower rate than in the shell (data not shown). There are also differences in the pattern of dopaminergic responses in the shell and the core of the NAc to cues that predict cocaine delivery (Aragona et al., 2009). Thus, while 
it is clear that dopaminergic responses in the core and shell differ during learning, the functional meaning of these differences is still unclear.

Here, we show that the amplitude of cue-evoked dopamine release in the NAc shell reflects salient information about predicted reinforcer magnitude. In our experimental design the audio-visual cue presented in all three of the reinforcer magnitude conditions was identical. Thus, the same audio-visual cue was able to elicit differential amounts of dopamine release based on the magnitude of the reinforcer it predicted. The ability of the same cue to exhibit differential dopamine concentrations related to its association with reward magnitude may be related to the power of ICS as a reinforcer. Regardless, our findings clearly demonstrate that dopaminergic transmission in the NAc shell is highly adaptable in the encoding of important information about future rewarding events, particularly reward magnitude.

An alternative explanation for the differential changes in cue-evoked dopamine release as a function of reinforcer magnitude may be that the observed fluctuations in cue-evoked dopamine release were strictly a physiological function of the electrical stimulations delivered. For example, in the high reinforcer magnitude condition, where a high stimulation current was delivered, it is possible that the intensity of the stimulation applied left the neurons in a more excitable state. Thus, in the trial immediately following each high amplitude stimulation, neurons could have an increased propensity to fire in response to cue onset, resulting in higher amplitudes of cue-evoked dopamine release. To investigate this possibility, we examined the relationship between cue-evoked and stimulated dopamine release in each reinforcer magnitude condition. If cue-evoked dopamine were solely a physiological function of the electrical stimulation, one would expect it to increase similarly to stimulated release. However, this was not the case. The slopes of the lines through cueevoked and stimulated dopamine release values for all three magnitudes were significantly different from one another (Figure 4B). Other potential confounds with the block design we used include within block learning or variations in arousal. However, these aspects were not apparent in the experimental results because dopamine responses to the cue remained essentially constant during a session with a single reward magnitude. Thus, we believe that the changes in the amplitude of cue-associated dopamine transients seen here reflect changes in reward value rather than a simple physiological linking of the two sets of dopamine release events.

We have previously shown that cue-evoked dopamine release in the NAc shell during ICSS is dependent on phasic activation of dopamine neurons in the VTA (Sombers et al., 2009). Midbrain dopamine neurons have been shown to report reward prediction errors such that when a reward is better than predicted, the dopaminergic response is positive and an increase in firing occurs (Schultz et al., 1997). Conversely, when a reward is worse than predicted (or is omitted altogether) the dopaminergic response is negative and a depression in firing occurs. Consistent with this, it has been found that the degree of phasic activation in midbrain dopamine neurons varied monotonically with reinforcer magnitude (Tobler et al., 2005). Similarly, cues that predict larger reinforcer magnitudes produce greater activation of dopamine neurons than do cues predicting smaller reinforcer magnitudes (Tobler et al., 2005). Our results indicate that dopamine release in the NAc shell reflects these changes in neural firing, as cues that predicted higher reinforcer magnitudes consistently produced greater amplitudes of dopamine release than cues that predicted smaller reinforcer magnitudes. Our results are similar to those reported in the NAc core for food reward (Gan et al., 2010). Taken together, these results give an unequivocal view that phasic dopaminergic transmission in the NAc shell acts as a sensitive measure of reinforcer magnitude. Future studies are needed to determine if dopamine release across other terminal regions (e.g., dorsal striatum) similarly track reinforcer magnitude or if this action is unique to the NAc. 


\section{Acknowledgments}

The authors thank Robert Wheeler, Catarina Owesson-White, Jennifer Ariansen and the UNC Department of Chemistry Electronics Facility. This work was supported by DA 10900 (RMW \& RMC) and DA 17318 (RMC \& RMW).

\section{Comprehensive list of Abbreviations"}

$\begin{array}{ll}\text { ICSS } & \text { intracranial self stimulation } \\ \text { NAc } & \text { nucleus accumbens } \\ \text { SEM } & \text { standard error of the mean } \\ \text { VTO } & \text { variable time-out }\end{array}$

\section{References}

Aragona BJ, Day JJ, Roitman MF, Cleaveland NA, Wightman RM, Carelli RM. Regional specificity in the real-time development of phasic dopamine transmission patterns during acquisition of a cuecocaine association in rats. Eur J Neurosci. 2009; 30:1889-1899. [PubMed: 19912327]

Arvanitogiannis A, Shizgal P. The reinforcement mountain: allocation of behavior as a function of the rate and intensity of rewarding brain stimulation. Behav Neurosci. 2008; 122:1126-1138. [PubMed: 18823168]

Cheer JF, Aragona BJ, Heien ML, Seipel AT, Carelli RM, Wightman RM. Coordinated accumbal dopamine release and neural activity drive goal-directed behavior. Neuron. 2007; 54:237-244. [PubMed: 17442245]

Corbett D, Wise RA. Intracranial self-stimulation in relation to the ascending dopaminergic systems of the midbrain: a moveable electrode mapping study. Brain Res. 1980; 185:1-15. [PubMed: 7353169]

Day JJ, Roitman MF, Wightman RM, Carelli RM. Associative learning mediates dynamic shifts in dopamine signaling in the nucleus accumbens. Nat Neurosci. 2007; 10:1020-1028. [PubMed: 17603481]

Dayan P, Balleine BW. Reward, motivation, and reinforcement learning. Neuron. 2002; 36:285-298. [PubMed: 12383782]

Fiorillo CD, Tobler PN, Schultz W. Discrete coding of reward probability and uncertainty by dopamine neurons. Science. 2003; 299:1898-1902. [PubMed: 12649484]

Gallistel CR, Leon M. Measuring the subjective magnitude of brain stimulation reward by titration with rate of reward. Behav Neurosci. 1991; 105:913-925. [PubMed: 1663762]

Gallistel CR, Leon M, Waraczynski M, Hanau MS. Effect of current on the maximum possible reward. Behav Neurosci. 1991; 105:901-912. [PubMed: 1663761]

Gan JO, Walton ME, Phillips PE. Dissociable cost and benefit encoding of future rewards by mesolimbic dopamine. Nat Neurosci. 2010; 13:25-27. [PubMed: 19904261]

Goto Y, Grace AA. Dopaminergic modulation of limbic and cortical drive of nucleus accumbens in goal-directed behavior. Nat Neurosci. 2005; 8:805-812. [PubMed: 15908948]

Heien ML, Johnson MA, Wightman RM. Resolving neurotransmitters detected by fast-scan cyclic voltammetry. Anal Chem. 2004; 76:5697-5704. [PubMed: 15456288]

Heien ML, Khan AS, Ariansen JL, Cheer JF, Phillips PE, Wassum KM, Wightman RM. Real-time measurement of dopamine fluctuations after cocaine in the brain of behaving rats. Proc Natl Acad Sci U S A. 2005; 102:10023-10028. [PubMed: 16006505]

Hollerman JR, Schultz W. Dopamine neurons report an error in the temporal prediction of reward during learning. Nat Neurosci. 1998; 1:304-309. [PubMed: 10195164]

Hyland BI, Reynolds JN, Hay J, Perk CG, Miller R. Firing modes of midbrain dopamine cells in the freely moving rat. Neuroscience. 2002; 114:475-492. [PubMed: 12204216]

Ikemoto S. Dopamine reward circuitry: Two projection systems from the ventral midbrain to the nucleus accumbens-olfactory tubercle complex. Brain Res Rev. 2007 
Kita JM, Parker LE, Phillips PE, Garris PA, Wightman RM. Paradoxical modulation of short-term facilitation of dopamine release by dopamine autoreceptors. J Neurochem. 2007; 102:1115-1124. [PubMed: 17663751]

Kobayashi S, Schultz W. Influence of reward delays on responses of dopamine neurons. J Neurosci. 2008; 28:7837-7846. [PubMed: 18667616]

Montague PR, McClure SM, Baldwin PR, Phillips PE, Budygin EA, Stuber GD, Kilpatrick MR, Wightman RM. Dynamic gain control of dopamine delivery in freely moving animals. J Neurosci. 2004; 24:1754-1759. [PubMed: 14973252]

Morris G, Nevet A, Arkadir D, Vaadia E, Bergman H. Midbrain dopamine neurons encode decisions for future action. Nat Neurosci. 2006; 9:1057-1063. [PubMed: 16862149]

Nicola SM, Taha SA, Kim SW, Fields HL. Nucleus accumbens dopamine release is necessary and sufficient to promote the behavioral response to reward-predictive cues. Neuroscience. 2005; 135:1025-1033. [PubMed: 16165291]

Olds J, Milner P. Positive reinforcement produced by electrical stimulation of septal area and other regions of rat brain. J Comp Physiol Psychol. 1954; 47:419-427. [PubMed: 13233369]

Olds ME, Olds J. Approach-avoidance analysis of rat diencephalon. J Comp Neurol. 1963; 120:259295. [PubMed: 13939939]

Owesson-White CA, Cheer JF, Beyene M, Carelli RM, Wightman RM. Dynamic changes in accumbens dopamine correlate with learning during intracranial self-stimulation. Proc Natl Acad Sci U S A. 2008; 105:11957-11962. [PubMed: 18689678]

Paxinos, G.; Watson, C. The Rat Brain in Stereotaxic Coordinates. Academic Press; New York: 2007.

Phillips PE, Robinson DL, Stuber GD, Carelli RM, Wightman RM. Real-time measurements of phasic changes in extracellular dopamine concentration in freely moving rats by fast-scan cyclic voltammetry. Methods Mol Med. 2003a; 79:443-464. [PubMed: 12506716]

Phillips PE, Stuber GD, Heien ML, Wightman RM, Carelli RM. Subsecond dopamine release promotes cocaine seeking. Nature. 2003b; 422:614-618. [PubMed: 12687000]

Robinson DL, Venton BJ, Heien ML, Wightman RM. Detecting subsecond dopamine release with fast-scan cyclic voltammetry in vivo. Clin Chem. 2003; 49:1763-1773. [PubMed: 14500617]

Roesch MR, Calu DJ, Schoenbaum G. Dopamine neurons encode the better option in rats deciding between differently delayed or sized rewards. Nat Neurosci. 2007; 10:1615-1624. [PubMed: 18026098]

Roitman MF, Stuber GD, Phillips PE, Wightman RM, Carelli RM. Dopamine operates as a subsecond modulator of food seeking. J Neurosci. 2004; 24:1265-1271. [PubMed: 14960596]

Sax L, Gallistel CR. Characteristics of spatiotemporal integration in the priming and rewarding effects of medial forebrain bundle stimulation. Behav Neurosci. 1991; 105:884-900. [PubMed: 1663760]

Schultz W, Dayan P, Montague PR. A neural substrate of prediction and reward. Science. 1997; 275:1593-1599. [PubMed: 9054347]

Simmons JM, Gallistel CR. Saturation of subjective reward magnitude as a function of current and pulse frequency. Behav Neurosci. 1994; 108:151-160. [PubMed: 8192841]

Simon H, Le Moal M, Cardo B. Self-stimulation in the dorsal pontine tegmentum in the rat. Behav Biol. 1975; 13:339-347. [PubMed: 1091258]

Sombers LA, Beyene M, Carelli RM, Wightman RM. Synaptic overflow of dopamine in the nucleus accumbens arises from neuronal activity in the ventral tegmental area. J Neurosci. 2009; 29:17351742. [PubMed: 19211880]

Tobler PN, Fiorillo CD, Schultz W. Adaptive coding of reward value by dopamine neurons. Science. 2005; 307:1642-1645. [PubMed: 15761155]

Waraczynski MA. The central extended amygdala network as a proposed circuit underlying reward valuation. Neurosci Biobehav Rev. 2006; 30:472-496. [PubMed: 16243397]

Waraczynski MA, Kaplan JM. Frequency-response characteristics provide a functional separation between stimulation-bound feeding and self-stimulation. Physiol Behav. 1990; 47:843-851. [PubMed: 2388940]

Wise RA. Addictive drugs and brain stimulation reward. Annu Rev Neurosci. 1996; 19:319-340. [PubMed: 8833446] 
Wise RA. Dopamine, learning and motivation. Nat Rev Neurosci. 2004; 5:483-494. [PubMed: 15152198] 


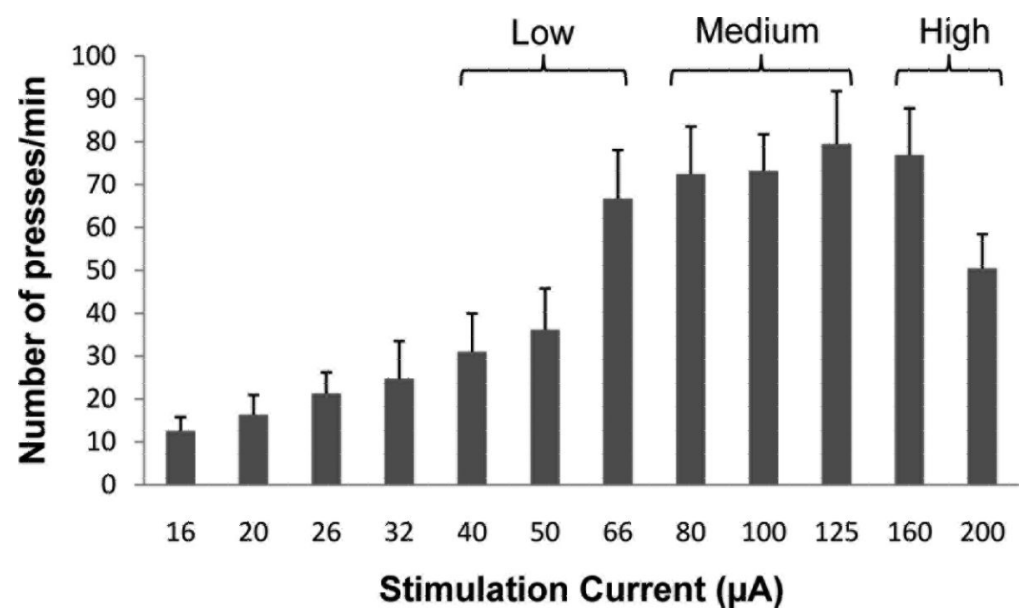

Figure 1. Reinforcer magnitude influences response rates for ICSS

The threshold curve shows the average number of presses in a $1 \mathrm{~min}$ interval for a range of stimulation currents ( $\mathrm{n}=6$ animals). Based on these findings, the average currents chosen for the low, medium and high reinforcer magnitudes were $67 \mu \mathrm{A} \pm 8.71,115 \mu \mathrm{A} \pm 11.4$, and $186.67 \mu \mathrm{A} \pm 8.43$, respectively (see methods for details). 

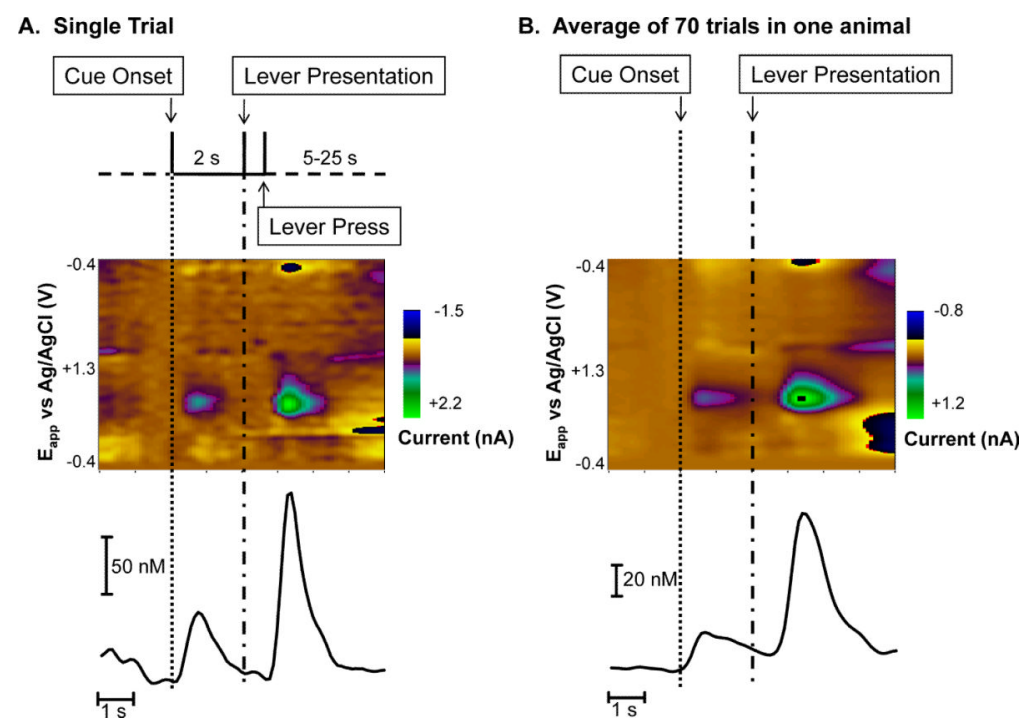

Figure 2. Transient increases in dopamine release follow cue onset during ICSS

A) Top, A time line of the ICSS paradigm used during dopamine measurements. Each trial began with cue onset ( 2 s prior to lever extension) and ended with stimulation delivery.

Trials were separated by a random interval of $5 \mathrm{~s}-25 \mathrm{~s}$ (as indicated by the dashed line). Center, a color plot from a single trial in a single animal. It displays the transient dopamine activity observed in response to cue onset and stimulation. Changes in current due to dopamine oxidation are color-coded and occur at the oxidation potential for dopamine $(\sim 0.6$ $\mathrm{V}$ vs. $\mathrm{Ag} / \mathrm{AgCl}$ ). Bottom, the dopamine concentration extracted from these data using principal component regression are shown. B) A color plot and corresponding concentration versus time trace averaged over 70 trials during responding for a high stimulation current in a single animal. 
A.

A. Low

Medium

High
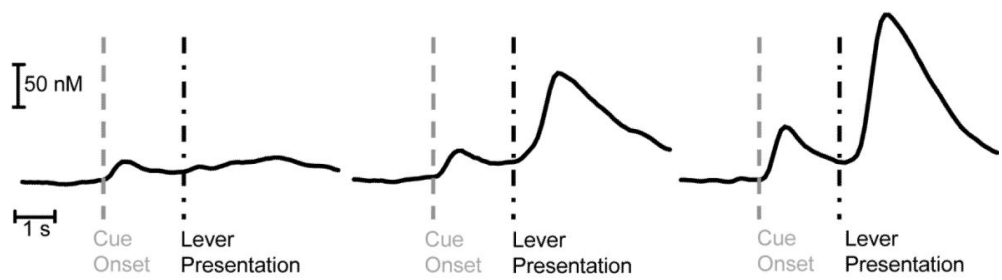

B.
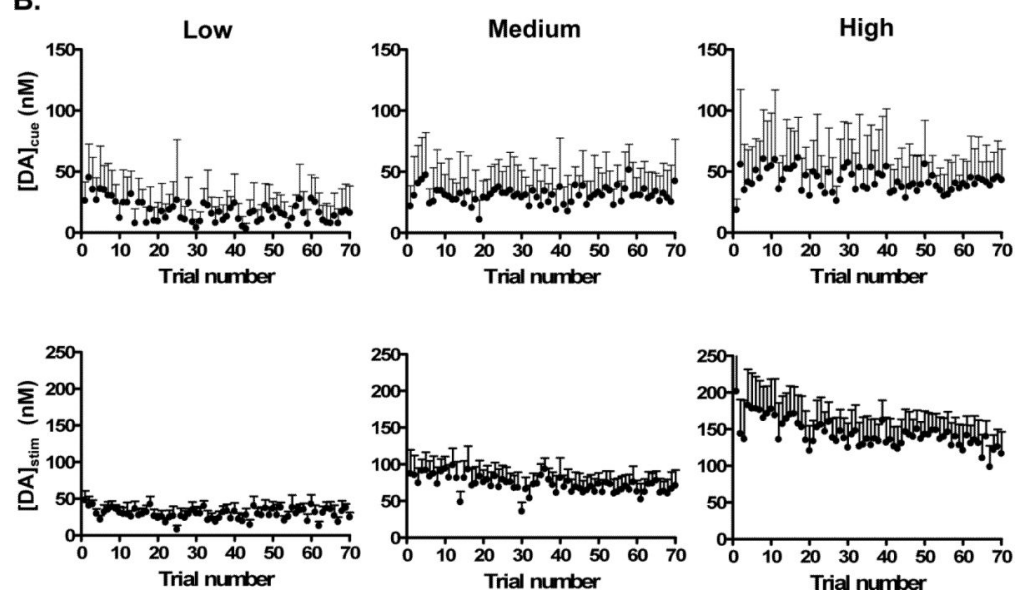

Figure 3. Cue-evoked dopamine release in the NAc shell varies as a function of reinforcer magnitude

A) Representative dopamine concentration traces are shown for the low, medium, and high reinforcer magnitude conditions. Cue onset is indicated by the first dashed line (gray) and lever presentation is indicated by the second dashed line (black). B) Average concentrations across each trial for cue-evoked (upper) and stimulated-evoked (lower) dopamine at different reinforcer magnitudes. Data points are the average $( \pm$ SEM $)$ across animals $(n=6)$. 
A.

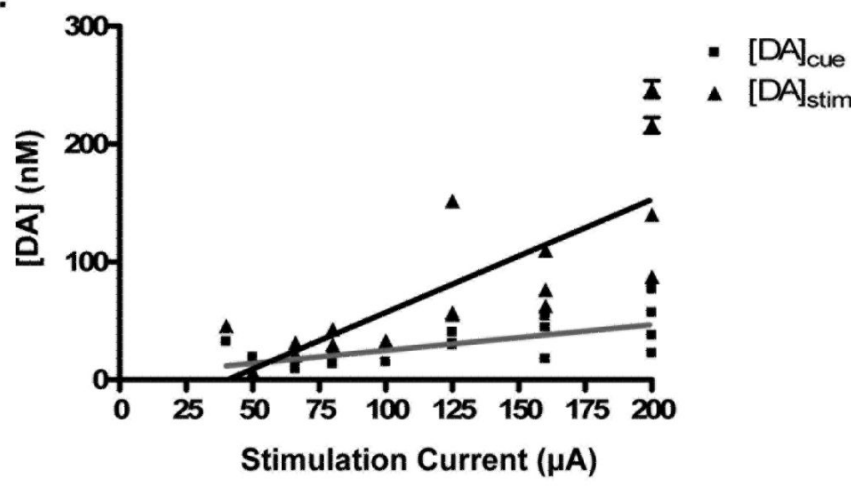

B.

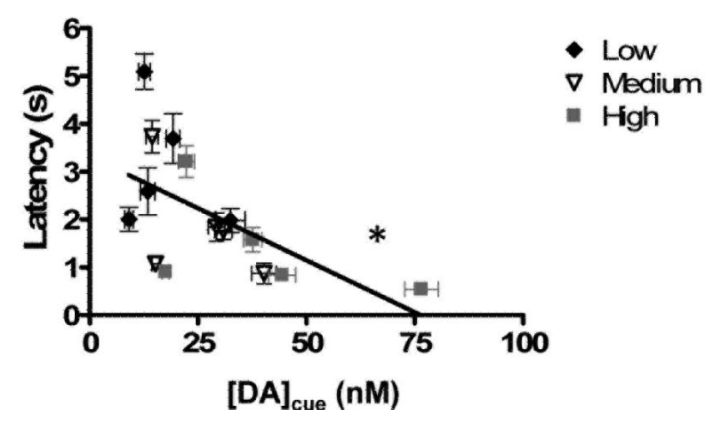

Figure 4. Cue-evoked dopamine in the NAc shell significantly influences reward-seeking behavior and is a function of reinforcer value

A) The slopes of the average cue-evoked (gray line) and stimulated (black line) dopamine signals as a function of stimulation current were significantly different from one another ( $\mathrm{p}$ $<0.001)$. The slopes were obtained from a linear regression analysis. B) Cue-evoked dopamine was significantly correlated with the latency to press in an inverse manner $\left(\mathrm{r}^{2}=\right.$ $\left.0.337,{ }^{*} \mathrm{p}<0.05\right)$. Each data point is the average response to the trials with low, medium or high ICSS currents from each animal. 
Table 1

Reinforcer magnitude significantly affects dopamine release in the NAc shell and the average latency to lever press

The amplitude of cue-evoked and electrically-stimulated dopamine release varied as a function of reinforcer magnitude $(H>M>L)$. Increases in reinforcer magnitude also lead to a decrease in the average latency to lever press $(\mathrm{H}<\mathrm{M}<\mathrm{L})$

\begin{tabular}{|c|c|c|c|}
\hline Reinforcer magnitude & Cue-evoked dopamine (nM) & Stimulated dopamine (nM) & Latency to Lever Press (s) \\
\hline Low & $15.7 \pm 0.9$ & $29.4 \pm 0.9$ & $2.9 \pm 0.91$ \\
\hline Medium & $30.2 \pm 0.8^{* * *}$ & $72.9 \pm 1.4^{* * *}$ & $1.8 \pm 0.11^{* * *}$ \\
\hline High & $42.1 \pm 1.1^{* * *}$ & $144 \pm 2.2^{* * *}$ & $1.4 \pm 0.10^{*}$ \\
\hline
\end{tabular}

